\title{
Dermatologists' Role in the Diagnosis and Management of Coronavirus Disease 2019 Coagulopathy
}

\author{
Payal Shah ${ }^{1} \cdot$ Kristen Lo Sicco $^{1} \cdot$ Avrom S. Caplan $^{1} \cdot$ Alisa N. Femia ${ }^{1} \cdot$ John G. Zampella ${ }^{1}$
}

Published online: 9 July 2020

C) Springer Nature Switzerland AG 2020

Evidence of coagulopathy in coronavirus disease 2019 (COVID-19) is associated with increased morbidity and mortality, especially in patients with severe disease. The cutaneous signs of COVID-19 can manifest this hypercoagulable state, creating an important role for dermatologists in the diagnosis and management of this new disease. The pathophysiology of coagulopathy in COVID-19 involves dysregulation of angiotensin converting enzyme 2 (ACE2)the protein attachment site of SARS-CoV-2 expressed in alveolar cells, cardiac myocytes, and vascular endothelium. The dysregulation of ACE2, systemic inflammatory host response, and the direct viral effects of SARS-CoV-2 leads to endothelial damage and stasis, creating a prothrombotic state in patients with active and possibly recent infection.

Cutaneous signs of COVID-19 in Europe have described the spectrum of dermatologic manifestations, with inflammatory lesions predominating over vascular $[1,2]$. The vascular subset has diverse morphologies, including violaceous porcelain-like macules, livedo, non-necrotic purpura, retiform purpura, chilblains, and Raynaud's phenomenon. Additionally, cases of acro-ischemia have been reported, presenting as violaceous plaques, vesicobullae, or dry gangrene [3]. The coagulopathy of COVID-19 likely contributes to the underlying pathophysiology of these vascular lesions. In fact, histopathologic examination of livedo and retiform purpura have exhibited pauci-inflammatory thrombogenic vasculopathy, with deposition of C5b-9 and C4d in affected and normal skin, similar to pathology seen in the lungs [4]. While chilblain-like lesions have been commonly reported among untested patients for COVID-19, the exact etiology

Payal Shah

Payal.shah@nyulangone.org

John G. Zampella

john.zampella@nyulangone.org

1 The Ronald O. Perelman Department of Dermatology, New York University Grossman School of Medicine, 240 E 38th St 14th floor, New York, NY 10016, USA remains unknown. Differences between children and adults may provide a clue towards pathophysiology. Morphologies of the pediatric populations range from a greater predominance of chilblain-like lesions, demonstrating an idiopathic lymphocytic infiltrate and no evidence of thrombosis or vasculitis, to a recent description of a Kawasaki-like illness, underscoring the risk of vascular damage [3]. In contrast, adults manifest more livedo and necrosis, suggesting a microthrombotic or vasculitic mechanism [1]. More evidence is needed to clarify the cutaneous signs that involve thromboembolic mechanisms.

On autopsy specimen examination of COVID-19 patients, microvascular thrombi have been found within the lungs and other organs, including the skin, underscoring the critical role of coagulopathy in precipitating COVID-19 mortality via tissue ischemia and multi-organ failure. Among COVID19-positive patients, emerging evidence suggests increased risk of venous thromboembolism (VTE), in the form of deep vein thrombosis or pulmonary emboli, and arterial thrombosis, in the form of myocardial infarction (MI) and strokes. In fact, both VTE and strokes have been reported as presenting symptoms of COVID-19. Even after receiving standard dosing of thromboprophylaxis, COVID-19-positive patients in the intensive care unit continue to have complications of VTE (27\%) and arterial thrombosis (3.7\%), evidencing the hypercoagulable nature of the disease [5]. Moreover, the induction of antiphospholipid antibodies may further potentiate thrombotic risk in the COVID-19 population. Although the correlation between skin manifestations of COVID-19 coagulopathy and severity of disease has not yet been elucidated, these cutaneous signs may herald life-threatening thrombosis and prognosticate a poor clinical outcome.

As more cutaneous manifestations of COVID-19 are reported, dermatologists have a crucial role in characterizing the association between SARS-CoV-2 infection and hypercoagulability, as well as predicting and preventing life-threatening thrombotic events. Current guidelines recommend therapeutic anticoagulation for severe COVID-19 
inpatients. More recently, consideration for extended treatment post-discharge with betrixaban, rivaroxaban, or aspirin in lower risk patients has also been recommended by the American Society of Hematology. As outpatient clinical encounters for vasculopathic eruptions increase, more efforts will be needed to help guide the evaluation, prevention, and treatment of hypercoagulability in post-discharge or non-hospitalized patients. In this setting, the baseline index of suspicion for thrombotic events in COVID-19 patients should be elevated, with cutaneous signs of hypercoagulability prompting close follow-up. In adult patients with new onset livedo reticularis, chilblain-like lesions, retiform purpura, and acral ischemia, COVID-19 diagnostic testing and skin biopsy should be considered to help characterize these cutaneous signs. Clinical evidence has demonstrated that serum markers of inflammation track with COVID-19 severity, suggesting that laboratory monitoring of disease severity may help prevent a poor outcome. For COVID-19-positive patients in the outpatient setting, particularly those with evidence of microthrombosis on physical examination, further evaluation of coagulation and vascular inflammation with laboratory tests, including platelets, antiphospholipid antibodies, D-dimers, fibrinogen, prothrombin time (PT), partial thromboplastin time (PTT), erythrocyte sedimentation rate (ESR), and C-reactive protein (CRP), can be considered to help assess the risk of thrombosis and the benefit from antithrombotic therapy. In particular, patients at elevated baseline risk for thrombosis, such as those with a prior history of significant thrombotic events (VTE, MI, or stroke), malignancy, or recent hospitalization, with clinical evidence suggestive of coagulopathy, may require empiric treatment with anticoagulants or antiplatelets, or referral to primary care for further evaluation. Clinical investigations within dermatology characterizing the pathophysiology of these vascular cutaneous signs of
COVID-19, stratifying thrombotic risk of patients with cutaneous signs of coagulopathy, and elucidating the risk-benefit profile of antiplatelet or anticoagulation therapy in these patients are warranted as an important next step to advance our diagnosis and management of COVID-19.

Funding No funding was received for the preparation of this manuscript.

\section{Compliance with ethical standards}

Conflicts of Interest The authors have no conflicts of interest to declare.

\section{References}

1. Galván Casas C, Català A, Carretero Hernández G, et al. Classification of the cutaneous manifestations of COVID-19: a rapid prospective nationwide consensus study in Spain with 375 cases. Br J Dermatol. 2020. https://doi.org/10.1111/bjd.19163 (Epub 29 Apr 2020).

2. Bouaziz JD, Duong T, Jachiet M, et al. Vascular skin symptoms in COVID-19: a French observational study. J Eur Acad Dermatol Venereol. 2020. https://doi.org/10.1111/jdv.16544 (Online ahead of print).

3. Cordoro KM, Reynolds SD, Wattier R, McCalmont TH. Clustered cases of acral perniosis: clinical features, histopathology and relationship to COVID-19. Pediatr Dermatol. 2020;37(3):419-23. https://doi.org/10.1111/pde.14227.

4. Magro C, Mulvey JJ, Berlin D, et al. Complement associated microvascular injury and thrombosis in the pathogenesis of severe COVID-19 infection: a report of five cases. Transl Res. 2020;220:1-13. https://doi.org/10.1016/j.trs1.2020.04.007.

5. Klok FA, Kruip M, van der Meer NJM, et al. Incidence of thrombotic complications in critically ill ICU patients with COVID-19. Thromb Res. 2020;191:145-7. https://doi.org/10.1016/j.throm res.2020.04.013. 